\title{
BMJ Open Barriers to the psychological well-being of Australian junior doctors: a qualitative analysis
}

\author{
Malcolm Peter Forbes, ${ }^{\oplus 1}$ Sweatha lyengar, ${ }^{2}$ Margaret Kay ${ }^{3}$
}

To cite: Forbes MP, lyengar S, Kay M. Barriers to the psychological well-being of Australian junior doctors: a qualitative analysis. BMJ Open 2019;9:e027558. doi:10.1136/ bmjopen-2018-027558

- Prepublication history for this paper is available online. To view these files, please visit the journal online (http://dx.doi. org/10.1136/bmjopen-2018027558).

Received 30 0ctober 2018 Revised 22 May 2019 Accepted 23 May 2019
Check for updates

(C) Author(s) (or their employer(s)) 2019. Re-use permitted under CC BY-NC. No commercial re-use. See rights and permissions. Published by BMJ.

${ }^{1}$ Department of Psychiatry, Faculty of Medicine, Dentistry and Health Sciences, University of Melbourne, Melbourne, Victoria, Australia

${ }^{2}$ Faculty of Medicine and Biomedical Sciences, The University of Queensland, Brisbane, Queensland, Australia ${ }^{3}$ Primary Care Clinical Unit, The University of Queensland, Brisbane, Queensland, Australia

Correspondence to Dr Malcolm Peter Forbes; malcolmpforbes@gmail.com

\section{ABSTRACT}

Objective To explore factors associated with the psychological well-being of junior doctors in Australia. Design Qualitative study using semistructured interviews. Setting Three teaching hospitals in Brisbane, Queensland, Australia.

Participants Fifteen junior medical officers (postgraduate year 2 doctors) employed across three hospitals in

Queensland participated in the study.

Main outcome measures Fifteen de-identified interviews were analysed. Four key themes emerged-workplace issues impacting on health and well-being; experiences of bullying and harassment; strategies to improve health and well-being; and barriers to seeking healthcare.

Conclusion Underlying system and cultural factors affect the health of junior doctors. Self-stigma particularly affects junior doctors and impacts on their healthcare seeking behaviours.

\section{INTRODUCTION}

Junior doctors in Australia experience significant psychological distress. The reasons for this are multifactorial. ${ }^{1}$ High rates of exhaustion, cynicism and compassion fatigue in this population place junior doctors at high risk of burnout ${ }^{2}{ }^{3}$ and depression. ${ }^{4}$ To address this, systematic reviews have supported the introduction of well-being initiatives. ${ }^{5-7}$ However, there is a paucity of qualitative research investigating the underlying reasons for psychological distress and why, how and to what extent well-being initiatives are effective. This study sought to explore the perceived barriers to psychological well-being in junior doctors and to understand the impact of a recently introduced resilience and well-being programme for junior doctors, the Resilience on the Run (RoR) programme. This RoR programme was specifically designed for junior doctors aiming to enhance skills to improve doctors' resilience and well-being, reflecting evidence that suggests resilience-including self-efficacy, planning and persistence-can be enhanced through educational interventions. ${ }^{8}$ RoR sessions teach mindfulness, offer techniques for managing personal
Strengths and limitations of this study

Junior doctors in Australia experience relatively high rates of psychological distress but the underlying reasons for this are not yet fully understood.

- This study identifies unique workplace stressors that negatively impact on the psychological well-being of junior doctors.

- Better understanding of the workplace stressors affecting junior doctors will allow more targeted strategies to address workplace contributors to psychological distress.

and interpersonal workplace difficulties and provide information about how and where to seek professional support.

Doctors' well-being is intimately connected to the quality of care delivered to patients. In 2009, Wallace et al published a seminal paper in the Lancet that turned the spotlight on physician health, presenting it as a 'missing quality indicator'. ${ }^{9}$ Hence, a focus on enhancing junior doctor well-being can translate into improved outcomes for the doctor and for future patient care. ${ }^{10} 11$

Over the past three decades, the growing literature on doctors' health has mostly utilised quantitative methods. This approach fails to understand the contexts and underlying mechanisms that impact on outcomes. Qualitative data allow a richer understanding of the complex interplay between individual, workplace and cultural factors that impact on junior doctor well-being. It can facilitate identification of barriers to and enablers of well-being at a workplace and organisational level, providing an essential dimension to the evaluation of well-being interventions.

\section{METHODS}

All postgraduate year 2 junior doctors at Metro South Health (a network of four hospitals in South East Queensland) were invited by email to participate in the study. 
Participation was voluntary and written informed consent was obtained. Participants were not involved in the design of the study but contributed to recruitment through word of mouth. All potential participants who took up the offer to participate were accepted for the study, providing a convenience sample. Participants received a gift voucher for their participation in the study and were offered the transcript of their interview.

One-hour recorded interviews were conducted by a single researcher (MPF) with each of the participants, using a semistructured approach with an interview guide. The semistructured interview guide was developed following a systematic review of the literature ${ }^{12}$ and extensive consultation with junior doctors and doctors' health experts via representative bodies during the design of this study.

The guide included nine questions with a number of probes for each question. The guide focused on the questions and subsequent discussions of the topic but did not restrict the participant from providing unique insights or comments relevant to the topic. Participants were advised at the beginning of the interviews that there was no 'correct' response to any question and there was no expectation that they conform to any specific social or professional role but instead speak to their unvarnished personal experience. The interviewer (MPF) took field notes during each interview.

Five perspectives were explored in the semistructured interviews: (1) the health and well-being of junior doctors, (2) the role of senior doctors in enabling the health and well-being of junior doctors, (3) doctors' resilience at work, (4) issues around bullying and harassment by colleagues and patients and (5) perceptions about being a doctor-patient.

Recordings were de-identified for transcription. The transcripts were read in their entirety before coding. Two researchers (MPF and SI) independently coded and analysed the data by thematic analysis. One researcher was trained in grounded theory. Recurrent themes were identified using an iterative, inductive process. There were no disagreements between the researchers in identification of themes. As patterns emerged, common themes were grouped together into four categories. Divergent cases were noted, reported verbatim and discussed.

\section{Patient and public involvement}

This research was designed without patient or public involvement. The public were not invited to comment on the study design and were not consulted to develop relevant participant outcomes or interpret the results. Participants were offered a copy of their transcript but otherwise were not invited to contribute to the writing or editing of this document for readability or accuracy.

\section{RESULTS}

The 15 participants were aged between 24 and 32 years. Eight participants (53\%) were women. Less than half of the participants $(47 \%)$ reported having a general practitioner (GP). Most participants $(60 \%)$ had participated in the RoR programme provided during their intern educational training in 2016. While further data were collected on ethnicity, this has not been reported to protect anonymity of participants.

Four key themes were identified-workplace issues reported by junior doctors and their impact on health and well-being (table 1); experiences of bullying and harassment (table 2); strategies to improve the health and well-being of doctors (table 3); and barriers to seeking healthcare (table 4).

\section{Theme 1: Key workplace issues impacting on health and well- being}

All participants identified system and cultural factors that impact on the health and well-being of junior doctors. System issues, including workload and work hours, difficulty taking leave and discouragement to claim overtime, were identified by a number of participants as having a negative effect on health and well-being. After-hours shifts and certain rotations, in particular surgical rotations, were highlighted as particularly stressful.

The amount of things that you're expected to do in a day, you physically don't have enough time in your working day to do that and so that means that things get missed, you are not doing as good of a job as you'd like to. (A7, female)

Despite a contractual obligation for hospitals to provide a half an hour meal break to be taken during the day, participants frequently cited forgoing this meal break to attend to patient care or having their meal break interrupted by contact from nursing staff. Cultural factors were also identified as impacting on junior doctor's health, in particular direct or indirect pressure not to claim overtime. Participants outlined concerns about perceived incompetence and concerns about the impact of claiming overtime on their future career prospects (eg, missing out on a reference from their current supervisor for a future training position).

When you try to claim [overtime], you are seen as incompetent. (A10, male)

\section{Theme 2: Bullying and harassment concerns reported by junior doctors}

Several issues of bullying by colleagues emerged from participant interviews. Many participants reported instances of bullying and harassment from colleagues which they reported as an integral part of the culture of medicine, reflecting the natural hierarchy of medicine and the apprenticeship model of learning medicine. Bullying was perpetuated in all cases from more senior doctors to junior doctors. Participants described how this affected them personally, leading to feelings of disillusionment and inadequacy, with some participants 
Table 1 Key workplace issues reported by junior doctors and the impact they have on health and well-being

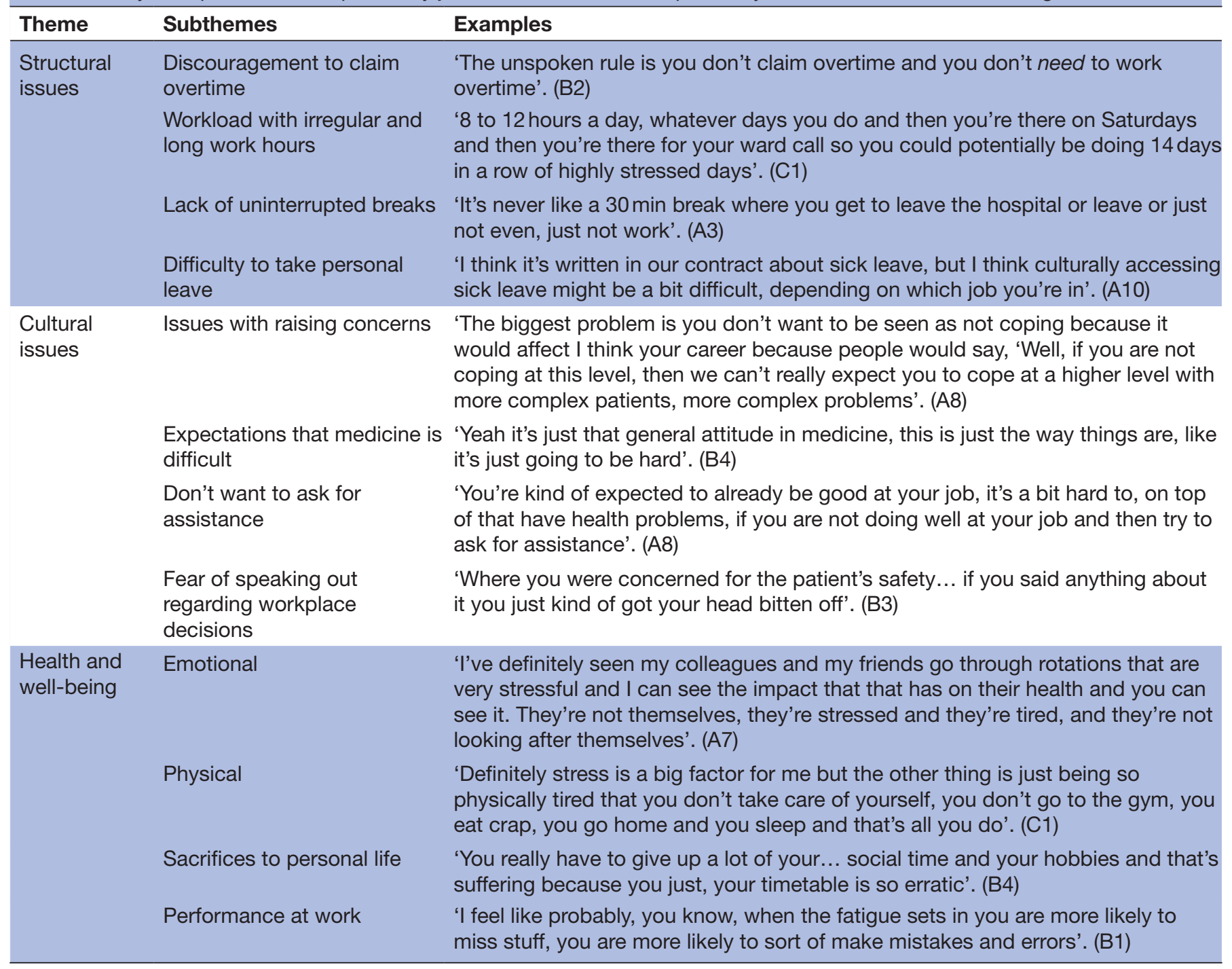

reporting not speaking up or asking questions for fear of being belittled.

It's an open secret amongst us; I mean it's something that we talk about. And we know that there are certain rotations and certain consultants to avoid. (A10, male)

It makes you feel like you're inadequate, which also I suppose means that you feel that you can't do a good enough job and maybe you're hesitant to do things that you should do. (A3, female)

Junior doctors also described bullying by other senior health professionals. This was reportedly most often from female senior nursing staff to female junior medical staff, as highlighted below:

If you say one wrong thing to a nurse, it will spread like wildfire and they'll put in all these complaints against you, but if a nurse, and often times a nurse does it, says something mean to you, you have to cop it. (C1, female)
Harassment, including reported sexual harassment and discrimination by patients, was also spoken about as being normalised within the culture of medicine. It appeared that harassment disproportionately affected female participants.

It seems like it's just the accepted way of behaving because that's how everyone else behaves. (C3, female)

Stuff like 'Oh, you Muslims are trouble and harassing everyone in the world and we're better off without you'... 'You should go back to where you came from'. (A3, female)

There was avoidance of complaining about doctors known to be bullies because of fear of retribution and fear it would impact on the junior doctor's career progression.

You'd feel like you don't want to make waves, rock the boat, make any complaints because it'll impact negatively on how you're viewed by that department. (B4, male) 
Table 2 Bullying and harassment concerns reported by junior doctors

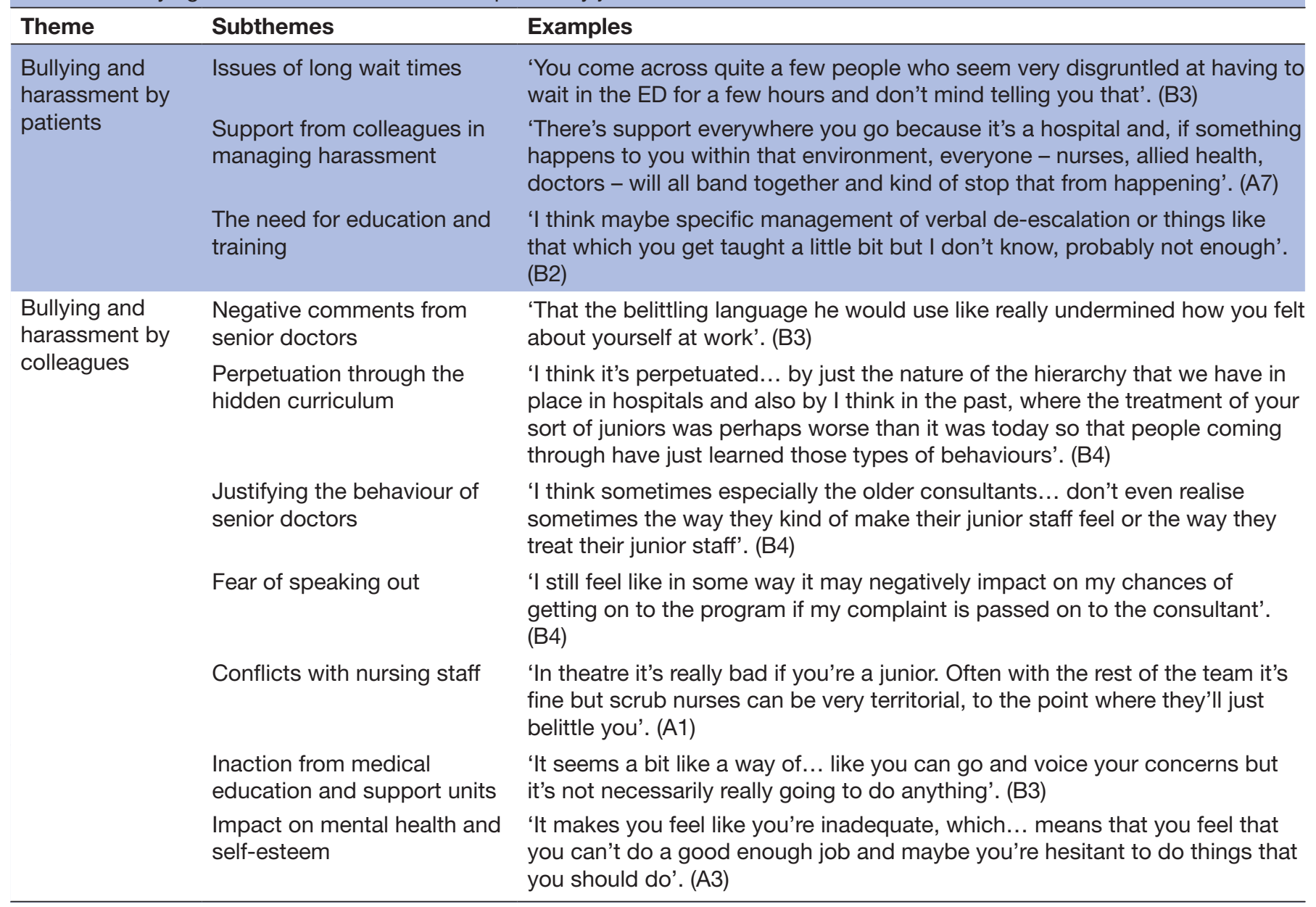

Theme 3: How to improve the health and well-being of doctors as identified by junior doctors

Participants recognised that senior doctors could be instrumental in empowering junior doctors by countering cultural norms in medicine and by role modelling good self-care. Senior doctors who were approachable, personable, empathetic and acknowledged the challenges of being a junior doctor contributed positively to the wellbeing of junior doctors.

I think that bosses have a role. [When working while physically unwell] Your bosses can turn a blind eye or they can say, 'You look shit. Go home.' (A10, male)

Bosses who are good leaders and good team members are the kinds of bosses who know when the workload is particularly high and some of them will come and sit (with junior doctors) and do discharge summaries.

(B3, female)

Just as a supportive supervisor could contribute to improved well-being, a preoccupied or uncaring supervisor appeared to have an equal but opposite effect. Participants reported their experience with senior doctors who were hierarchical, cold or aloof and how this led to feeling uncontained and unsupported on the ward. Participants reported that these supervisors appeared not to appreciate the changed nature of medicine, with shorter admissions and increased medico-legal and regulatory pressures leading to additional, time-consuming paperwork.

Some consultants would be so cold and that hierarchy of 'I'm up here and you're like a lesser being and you shouldn't ever talk to me' still exists. (A3, female)

I think most senior doctors would give the line of 'When I was a resident, we didn't have any of this so why should you?' (A3, female)

A number of doctors had undertaken RoR training as interns and this was appraised positively. Role-playing stressful clinical scenarios played a role in helping participants realise they were not alone in feeling stressed. Mindfulness training was considered interesting by some participants. However, resilience strategies were generally visualised as a limited strategy for promoting wellness and often an inadequate solution when significant stress or health problems already existed. This finding may have reflected different views on the definition of resilience and the purpose of resilience training.

They had a session where they took people's scenarios and then acted them out, it was good because 
Table 3 How to improve the health and well-being of doctors as identified by junior doctors

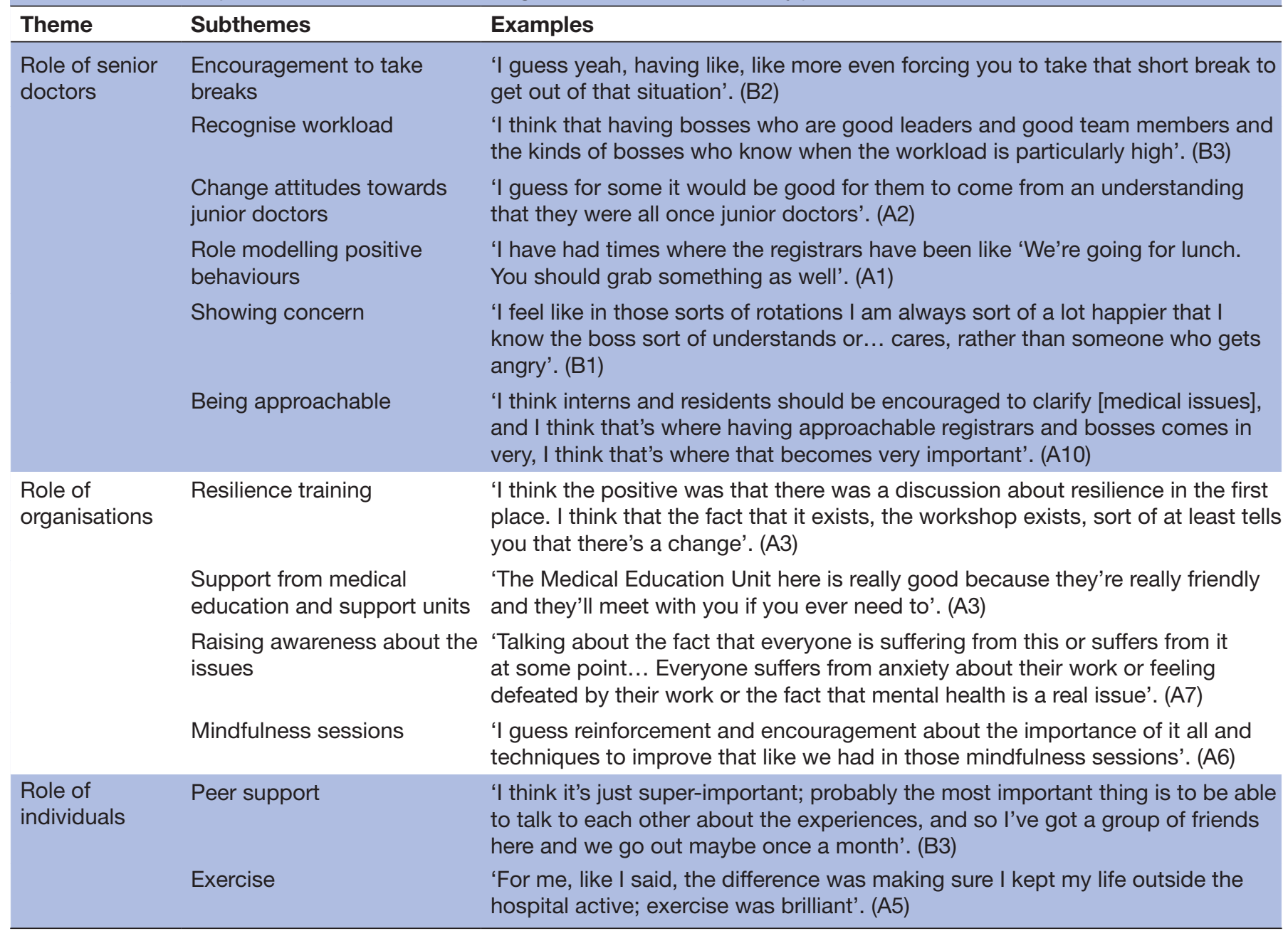

everyone could identify that 'I've been in that situation. Everyone has been in that situation.' (A1, female)

I think the resilience training strategies are good for maintenance of good health but, if someone is struggling, I think it's a band-aid over a gaping wound. (A5, male)

The conceptualisation of being a 'resilient doctor' varied between participants.

[Resilient doctors] can cope under pressure, they can bounce back and self-reflect and think about the mistakes that they've made and then improve from there. (A9, male)

It's not to say someone who is necessarily high functioning or doing well. I guess it has to do a lot with being able to take a hit and still remain at baseline. (A8, male)

Some doctors identified the challenges of being 'resilient' in a highly stressful environment. The culture of drinking to cope with stress was identified by a number of junior doctors.

\section{Theme 4: Barriers to seeking healthcare reported by junior} doctors

Junior doctors described barriers to becoming a 'doctor-patient'. Lack of time was a key barrier to health access. Stigma, both felt and enacted, was also important, especially when considering mental health issues.

I think there's still quite a bit of stigma about admitting you have a mental health problem... it's that sense of failure... whether it's depression or anxiety or whatever. (B4, female)

I think there's a stigma with regards to mental health but there's also that expectation that in our profession we are supposed to be resilient. (A2, female)

The concerns about stigma were magnified if there was a perceived impact on the junior doctor's career.

"You can't show any signs of weakness, especially if you're trying to get on the [specialty training] program or progressing through the program, trying to become a consultant'. (B1, male)

Cultural expectations also created barriers because the junior doctors felt that they should prioritise the needs 


\begin{tabular}{|c|c|c|}
\hline Theme & Subthemes & Examples \\
\hline \multirow[t]{3}{*}{$\begin{array}{l}\text { Barriers to } \\
\text { becoming a } \\
\text { doctor-patient }\end{array}$} & Embarrassment & $\begin{array}{l}\text { 'I think that's just kind of a general thing of doctors not wanting to look } \\
\text { stupid in front of another doctor, I mean especially ones who have kind of } \\
\text { specialised'. (B4) }\end{array}$ \\
\hline & Trivialising illness & $\begin{array}{l}\text { 'You just put it sort of further down the priorities because you know that it's } \\
\text { probably not going to kill you'. (B1) }\end{array}$ \\
\hline & Issues of time & $\begin{array}{l}\text { 'When you have a GP further away who might not work after hours and you're } \\
\text { working overtime, suddenly it's hard to even get an appointment'. (A5) }\end{array}$ \\
\hline $\begin{array}{l}\text { Concerns about } \\
\text { being a doctor- } \\
\text { patient }\end{array}$ & $\begin{array}{l}\text { Fear of lack of } \\
\text { confidentiality }\end{array}$ & $\begin{array}{l}\text { 'Probably for doctors' mental health, I think the biggest challenge would } \\
\text { probably be seeking help to begin with and actually knowing where to go to } \\
\text { talk about issues and just reinforcing their confidentiality and reinforcing that it } \\
\text { won't actually affect anything else in your life'. (A3) }\end{array}$ \\
\hline \multirow[t]{2}{*}{ Cultural issues } & Stigma of mental health & $\begin{array}{l}\text { 'In mental health, that's not like a visible illness, doctors themselves underplay } \\
\text { it because you can't see it, and it's put on the backfoot in that we don't see } \\
\text { GPs for those things'. (A3) }\end{array}$ \\
\hline & $\begin{array}{l}\text { Expectations that doctors } \\
\text { don't get sick }\end{array}$ & $\begin{array}{l}\text { 'No one thinks about seeking help because "I'm not that kind of person, I'm } \\
\text { not a depressed person. I'm fine. Look at me. I'm a doctor. I'm functioning", } \\
\text { (C1) }\end{array}$ \\
\hline
\end{tabular}

GP, general practitioner.

of their patients above their own at all times. In many cases, participants had minimised the seriousness of their health problems or neglected their own health to prioritise the health of patients and avoid letting down their colleagues by taking time off work when sick.

I think that we're all kind of taught to focus on patient health and that's a primary focus and that our health is probably secondary to it. I think that's ingrained in the culture of medicine and the idea of teaching, of telling people what to do, but not necessarily following through with what you're saying to other people. (A3, female)

Confidentiality when seeking healthcare was another issue that impacted on help-seeking behaviours.

I think there's a fear that there will be restrictions on your practising licence. (C1, female)

\section{DISCUSSION}

This study provides a deeper understanding of the factors associated with psychological distress in junior doctors in Australia. While many of the issues raised in this study have been described previously, this study is an important contribution as it provides greater insight about the various underlying factors that impact on psychological distress. Using qualitative methods, this study has captured a series of powerful narratives that clearly illustrate how system and cultural issues impact on the well-being of junior doctors. It is essential that interventions to improve junior doctor well-being first acknowledge the unique experiences of junior doctors. Quantitative measures of psychological distress cannot adequately inform the development of effective interventions because they do not signpost for whom an intervention is most likely to work and under what circumstances.

There are individual, system and cultural issues that impact on junior doctor health and well-being. It is known that personality factors and temperament can negatively impact on how doctors cope with the stressors of their work. ${ }^{13}$ However, hitherto adaptive personality traits can often be rendered maladaptive by a medical culture characterised by implicit or explicit demands for impeccable professionalism with limited room for vulnerability. ${ }^{14}$ As demonstrated in this study, many junior doctors feel they are not permitted to be sick or take time off. System factors, including lack of time due to long working hours, can contribute to delayed access to healthcare.

Junior doctors reported ingrained stigma with helpseeking behaviours, especially for mental health issues. Stigma attached to stress and mental illness was described as ubiquitous in the workplace. Both enacted stigma (discrimination against people experiencing stress or difficulty coping) and perceived stigma (the shame associated with experiencing stress or difficulty coping) were reported. Previous research with unwell doctors has 
also identified these concerns. In 2013, the beyondblue National Mental Health Survey of Doctors and Medical Students reported that $40.5 \%$ respondents believed that doctors with a history of mental health disorders were perceived as less competent by their peers and $44.8 \%$ believed that many doctors felt that experiencing a mental health condition was a sign of weakness'. ${ }^{2}$ Female doctors described greater discrimination and harassment in the workplace which resonates with previous Australian and international studies. ${ }^{215}$

A recent study exploring psychological distress in interns identified similar stressors intrinsic to the job such as work overload, long hours and unpaid overtime. ${ }^{16}$ Australian research has shown that $70 \%$ of overtime worked is not claimed by junior doctors and one of the key reasons relates to hospital culture, including the real or perceived pressure to under-claim or not claim extra hours worked. ${ }^{17}$ This study highlights the significant cultural issues that sustain the factors that negatively impact on junior doctors' health and well-being. Confidential disclosure of challenges and barriers disclosed in these individual interviews may have been suppressed in a group discussion.

Bullying, harassment and discrimination must be taken seriously in the medical profession. ${ }^{18}$ Workplace bullying is associated with an increased risk of depression and anxiety. ${ }^{19}$ Recent work by the Royal Australasian College of Surgeons to reduce bullying, discrimination and harassment will take time to have a positive impact while cultural factors continue to support poor behaviours. ${ }^{20}$ This study found that cultural expectations resulted in junior doctors tolerating unacceptable workplace behaviours that would be unlikely to be accepted in other work environments. Support by peers helped to validate their experiences with this solidarity acting as a coping strategy.

The participants in this study valued their resilience training during their intern year. This is consistent with previous reviews that identified the positive impact of diverse well-being interventions for junior doctors and medical students. ${ }^{56}$

The key limitations of this study include the potential bias in recruitment of participants. Junior doctors who experienced psychological stress may have been more likely to participate. The study focused on participants from one large hospital network but cannot be seen as representative of the experience of other hospitals. Junior doctors may have been more inclined to share this information because they knew that their hospitals had been focusing on health and well-being initiatives. This limits the generalisability of the findings. While there were common themes emerging strongly from these interviews, saturation was not assured with the limited participant numbers. However, the findings do resonate strongly with previous Australian and international studies.

Investing in the health and well-being of medicine's next generation of practitioners is a recognised priority. ${ }^{21}$ Better understanding of these stressors and the perception of these stressors is the first (and essential) step to identifying solutions to mitigate the individual, system and cultural factors associated with poor doctors' health. It is imperative that qualitative research methods are utilised to fully understand the problems and tailor interventions. The key to addressing psychological distress in the medical profession, both now and into the future, is early identification and early intervention.

Contributors MPF and MK conceptualised the study. MPF completed all interviews. MPF and SI completed data analysis. All authors contributed to writing the manuscript.

Funding Funding was provided through an Avant Mutual Doctor-in-Training Scholarship—value AUD \$12500.

Competing interests None declared.

Patient consent for publication Obtained.

Ethics approval Ethics approval was obtained from Metro South Human Research Ethics Committee (HREC/17/QPAH/48).

Provenance and peer review Not commissioned; externally peer reviewed. Data sharing statement The data are available to all authors of the study.

Open access This is an open access article distributed in accordance with the Creative Commons Attribution Non Commercial (CC BY-NC 4.0) license, which permits others to distribute, remix, adapt, build upon this work non-commercially, and license their derivative works on different terms, provided the original work is properly cited, appropriate credit is given, any changes made indicated, and the use is non-commercial. See: http://creativecommons.org/licenses/by-nc/4.0/.

\section{REFERENCES}

1. Forbes MP, Jenkins K, Myers MF. Optimising the treatment of doctors with mental illness. Aust N Z J Psychiatry 2019;53:106-8.

2. Beyondblue. National Mental Health Survey of Doctors and Medical Students. 2013. http://www.beyondblue.org.au/docs/default-source/ defaultdocument-library/bl1132-report-nmhdmss-full-report_web

3. Willcock SM, Daly MG, Tennant CC, et al. Burnout and psychiatric morbidity in new medical graduates. Med J Aust 2004;181:357-60.

4. Mata DA, Ramos MA, Bansal N, et al. Prevalence of depression and depressive symptoms among resident physicians: a systematic review and meta-analysis. JAMA 2015;314:2373-83.

5. West CP, Dyrbye LN, Erwin PJ, et al. Interventions to prevent and reduce physician burnout: a systematic review and meta-analysis. Lancet 2016;388:2272-81.

6. Clough BA, March S, Chan RJ, et al. Psychosocial interventions for managing occupational stress and burnout among medical doctors: a systematic review. Syst Rev 2017;6:144.

7. Petrie K, Crawford J, Baker STE, et al. Interventions to reduce symptoms of common mental disorders and suicidal ideation in physicians: a systematic review and meta-analysis. Lancet Psychiatry 2019;6:225-34.

8. Howe A, Smajdor A, Stöckl A. Towards an understanding of resilience and its relevance to medical training. Med Educ 2012;46:349-56.

9. Wallace JE, Lemaire JB, Ghali WA. Physician wellness: a missing quality indicator. Lancet 2009;374:1714-21.

10. Frank E, Dresner Y, Shani M, et al. The association between physicians' and patients' preventive health practices. CMAJ 2013;185:649-53.

11. Shanafelt TD, Balch CM, Bechamps G, et al. Burnout and medical errors among American surgeons. Ann Surg 2010;251:995-1000.

12. Forbes $M$, Byrom L, Manoharan B, et al. A systematic review of mental health and wellbeing programs for medical students and physicians. Boston, USA: International Conference on Physician Health, 2016:18-20.

13. Myers M, Gabbard GO. The Physician as Patient: A Clinical Handbook for Mental Health Professionals: American Psychiatric Publishing, 2008.

14. Stanton J, Randal P. Developing a psychiatrist-patient relationship when both people are doctors: a qualitative study. BMJ Open 2016;6:e010216.

15. Walton MM. Sexual equality, discrimination and harassment in medicine: it's time to act. Med J Aust 2015;203:167-9. 
16. Tallentire VR, Smith SE, Facey AD, et al. Exploring newly qualified doctors' workplace stressors: an interview study from Australia. BMJ Open 2017;7:e015890.

17. Forbes MP, Arthur T, Manoharan B, et al. Junior doctors and overtime: the Queensland experience. Intern Med J 2016;46:635-7.

18. Fnais $\mathrm{N}$, Soobiah $\mathrm{C}$, Chen $\mathrm{MH}$, et al. Harassment and discrimination in medical training: a systematic review and meta-analysis. Acad Med 2014;89:817-27.
19. Butterworth P, Leach LS, Kiely KM. Why it's important for it to stop: Examining the mental health correlates of bullying and ill-treatment at work in a cohort study. Aust N Z J Psychiatry 2016;50:1085-95.

20. Royal Australasian College of Surgeons. Expert Advisory Group on discrimination, bullying and sexual harassment. RACS 2015.

21. Baker K, Sen S. Healing Medicine's Future: Prioritizing Physician Trainee Mental Health. AMA J Ethics 2016;18:604-13. 